\title{
Bi-weekly eribulin therapy for metastatic breast cancer: a multicenter phase II prospective study (JUST-STUDY)
}

\author{
Shoichiro Ohtani ${ }^{1} \cdot$ Takahiro Nakayama $^{2} \cdot$ Tetsuhiro Yoshinami $^{2} \cdot$ Ken-ichi Watanabe $^{3} \cdot$ Fumikata Hara $^{4}$. \\ Yasuaki Sagara ${ }^{5} \cdot$ Hidetoshi Kawaguchi $^{6} \cdot$ Kenji Higaki $^{7} \cdot$ Nobuki Matsunami $^{8} \cdot$ Yoshie Hasegawa $^{9}$. \\ Masato Takahashi ${ }^{3} \cdot$ Makiko Mizutani $^{10} \cdot$ Takashi Morimoto $^{11} \cdot$ Masako Sato $^{3} \cdot$ Mitsuya Itoh $^{1} \cdot$ Satoshi Morita $^{12}$. \\ Norikazu Masuda ${ }^{10}$
}

Received: 31 August 2017 / Accepted: 31 January 2018 / Published online: 12 February 2018

(c) The Author(s) 2018. This article is an open access publication

\begin{abstract}
Background This study aimed to investigate whether schedule modification is safe and effective in patients intolerant to the standard eribulin dose and schedule.

Methods Patients with metastatic breast cancer (MBC) treated with both anthracycline and taxane and $\leq 3$ prior regimens of chemotherapy for $\mathrm{MBC}$ received eribulin at the standard dose and schedule $\left(1.4 \mathrm{mg} / \mathrm{m}^{2}\right.$ on days 1 and 8 of a 21 -day cycle) in the first cycle; change of dosing schedule $\left(1.4 \mathrm{mg} / \mathrm{m}^{2}\right.$ on days 1 and 15 of a 28-day cycle) was determined by change in neutrophil count, platelet count, aspartate aminotransferase, alanine aminotransferase, total bilirubin, serum creatinine, and non-hematological toxicity on day 8 of the first cycle or day 1 of the second cycle. Clinical benefit rate (CBR; primary endpoint), time to treatment failure (TTF), overall survival (OS), and safety were evaluated.

Results Of the 88 patients who were enrolled and received standard eribulin therapy in the first cycle, 42 patients were moved to the bi-weekly therapy group and 40 continued standard therapy. In the bi-weekly and standard therapy groups, mean relative dose intensity was 62.7 and $90.9 \%$, CBR was 31.0 and $25.0 \%$, median TTF was 81.5 and 75 days, and OS was 523 and 412 days, respectively. Neither group reported severe adverse events.

Conclusion This is the first study to show that a bi-weekly eribulin schedule is tolerable and has comparable efficacy in patients intolerant to the standard eribulin schedule.

Clinical trial registration University Hospital Medical Information Network (UMIN) Center (ID: UMIN 000008491).
\end{abstract}

Keywords Eribulin $\cdot$ Schedule modification $\cdot$ Metastatic breast cancer $\cdot$ Bi-weekly schedule

\section{Introduction}

Microtubule polymerization is a key process in cancer cell proliferation and a number of microtubule-targeting agents have been evaluated in preclinical and clinical studies [1]. Eribulin, an analog of halichondrin B, is a novel non-taxane microtubule dynamics inhibitor [2]. Because eribulin binds to a unique site on tubulin [3], it can be used to overcome taxane resistance and can be used in patients

Electronic supplementary material The online version of this article (https://doi.org/10.1007/s12282-018-0843-y) contains supplementary material, which is available to authorized users.

Shoichiro Ohtani

sho_ohtani@hotmail.com

Extended author information available on the last page of the article progressing after standard treatment with anthracyclineand taxane-containing regimens [4]. It has been approved in many countries for the treatment of metastatic breast cancer (MBC) in patients previously treated with chemotherapeutic agents like anthracycline- and taxane-based regimens [5-7]. In Japan, it is approved for the treatment of patients with inoperable or recurrent breast cancer [8] and is being investigated in patients who have not been previously treated with chemotherapy regimens for MBC [9].

Several clinical trials have demonstrated the antitumor activity and tolerability of eribulin. A global, multicenter, open-label, phase III, randomized study (EMBRACE study) demonstrated a significant and clinically meaningful improvement in overall survival (OS) with eribulin compared to that with treatment of physician's choice in patients with heavily pre-treated MBC (median OS 13.1 
vs. 10.6 months, $p=0.041$ ) [10]. In another randomized phase III study (301 study), median OS in the eribulin group was longer than that in the capecitabine group, though the results were not significantly different [15.9 vs. 14.5 months, hazard ratio (HR) 0.88 ; $95 \%$ confidence interval (CI) $0.77-1.00 ; p=0.056$ ] [11]. A pooled analysis of these two phase III studies demonstrated that eribulin significantly improved OS compared to control in various subgroups of patients with pretreated MBC [12]. A single-arm, multicenter, open-label phase II study conducted in Japan, which enrolled MBC patients pretreated with an anthracycline and a taxane, has also shown both the efficacy and tolerability of eribulin as first- to fourth-line treatment [13].

Although eribulin demonstrated a manageable safety profile, the incidence of myelosuppression is high. In the EMBRACE trial, adverse events occurred in $98.8 \%$ patients receiving eribulin [10]. The incidence of both neutropenia and leukopenia was $98.8 \%$ in the Japanese phase II study [13]. In the MBC population known to have low quality of life (QOL) [14], adverse events may have further unfavorable impact on patient well-being and may lead to discontinuation of the eribulin therapy especially in severe cases. A comparison of the clinical benefits of eribulin versus capecitabine using health-related QoL (HRQoL) data from a phase III randomized trial in patients with MBC showed similar impact on patient functioning with no overall difference in HRQoL; however, patients who received eribulin showed worse systemic side-effects of chemotherapy such as dry mouth, different tastes, irritated eyes, feeling ill, hot flushes, headaches, and hair loss and lesser gastrointestinal toxicity compared to those receiving capecitabine [15]. The main aims of MBC treatment are prolonging OS and maintaining QOL. While non-hematologic toxicity is lower with eribulin than with other chemotherapeutic agents, discontinuation due to hematologic toxicity is a major problem of eribulin therapy. Management of neutropenia is important to continue eribulin treatment for clinical benefits. Since the standard dosing schedule of eribulin often leads to severe neutropenia, the management of eribulin dose and schedule is a key issue to decrease toxicity and continue treatment. Dose reduction of eribulin has been reported for patients with adverse events of higher grades in the previous studies $[10,13]$. We conducted a phase II, non-randomized, prospective study to investigate whether schedule modification of bi-weekly eribulin therapy is safe and effective.

\section{Patients and methods}

\section{Patients}

Japanese women with MBC previously treated with an anthracycline and a taxane, and who had received up to three prior regimens of chemotherapy for MBC were included in the study. The main inclusion criteria were as follows: women aged $\geq 20$ years, histologically confirmed human epidermal growth factor receptor 2 (HER2)-negative breast cancer, Eastern Cooperative Oncology Group performance status (ECOG PS) [16] of 0-2, measurable lesion in at least one dimension by computed tomography or magnetic resonance imaging based on Response Evaluation Criteria in Solid Tumors (RECIST) ver. 1.1. [17], neutrophil count $\geq 1000 / \mu \mathrm{L}$, platelet count $\geq 100,000 / \mu \mathrm{L}$, hemoglobin $\geq 9.0 \mathrm{~g} / \mathrm{dL}$, aspartate aminotransferase and alanine aminotransferase $\leq 3.0$ times the upper limit of normal $(\mathrm{ULN})$ or $\leq 5.0 \times \mathrm{ULN}$ in patients with hepatic metastases, total bilirubin $\leq 1.5 \times$ ULN, serum creatinine $\leq 1.5 \times$ ULN, and expected survival of $\geq 3$ months.

Key exclusion criteria were as follows: systemic infection with fever $\geq 38.0^{\circ} \mathrm{C}$, pleural effusion, ascites or pericardial fluid requiring drainage, symptomatic brain metastasis, serious comorbidities (e.g., ischemic heart disease not controllable by treatment or heart disease such as arrhythmia, myocardial infarction $<6$ months prior to study entry, complication of interstitial pneumonia or pulmonary fibrosis), second active cancer, inadequately controlled diabetes mellitus, pregnancy, breastfeeding, or women with childbearing potential.

\section{Study design}

In the first cycle, $1.4 \mathrm{mg} / \mathrm{m}^{2}$ eribulin mesylate (equivalent to $1.23 \mathrm{mg} / \mathrm{m}^{2}$ of eribulin as free base) was administered intravenously over 2-5 min on days 1 and 8 of each 21-day cycle (the standard regimen). Based on the incidence of adverse events prior to eribulin administration on day 8 of the first cycle or day 1 of the second cycle, patients were allocated to standard therapy group or bi-weekly therapy group on day 1 of the second cycle. The criteria for dosing schedule modification included neutrophil count $\geq 1000 /$ $\mu \mathrm{L}$, platelet count $\geq 75,000 / \mu \mathrm{L}$, aspartate aminotransferase and alanine aminotransferase $\leq 3.0$ times the ULN or $\leq 5.0 \times$ ULN in patients with hepatic metastases, total bilirubin $\leq 1.5 \times \mathrm{ULN}$, serum creatinine $\leq 1.5 \times \mathrm{ULN}$, non-hematological toxicity with grade $\leq 2$. When patients met these criteria, they continued the standard regimen. But otherwise, they were allocated to the bi-weekly therapy, where $1.4 \mathrm{mg} / \mathrm{m}^{2}$ eribulin mesylate (equivalent to $1.23 \mathrm{mg} / \mathrm{m}^{2}$ of eribulin as free base) was administered on days 1 and 15 of each 28-day cycle. Eribulin was administered until disease progression, unacceptable toxicity, or withdrawal of consent. Table $\mathrm{S} 1$ shows the dosing schedule for patients switching to bi-weekly eribulin administration and requiring dose reduction and/or delayed administration. 
After patients were allocated to the two groups, they continued to receive eribulin treatment in the same group. Dose reduction to $1.1 \mathrm{mg} / \mathrm{m}^{2}$ was permitted to manage toxicity of grade $\geq 3$ febrile neutropenia, grade $\geq 3$ neutropenia with infection requiring antibiotic treatment, grade 4 thrombocytopenia, or grade $\geq 3$ non-hematological toxicity.

Eribulin administration was discontinued for patients requiring dose reduction to $<1.1 \mathrm{mg} / \mathrm{m}^{2}$ or delay in administration $>2$ weeks. Administration of granulocyte colonystimulating factor (G-CSF) was permitted in the event of grade 4 neutropenia or grade $\geq 3$ febrile neutropenia; however, the use of G-CSF for preventing neutropenia was not permitted.

\section{Rationale for dosing schedule modification}

Table S2 shows the theoretical dose intensity of eribulin in the standard schedule and bi-weekly schedule. When patients were unable to continue eribulin treatment on the standard regimen $\left(1.4 \mathrm{mg} / \mathrm{m}^{2}\right.$ on days 1 and 8 of each 21 -day cycle), the dose reduction to $1.1 \mathrm{mg} / \mathrm{m}^{2}$ on the same dosing schedule had been determined in the previous studies $[10,11]$ in which the dose intensity decreased to $0.73 \mathrm{mg} /$ $\mathrm{m}^{2}$ per week. A similar $\left(0.70 \mathrm{mg} / \mathrm{m}^{2}\right)$ dose intensity was maintained in bi-weekly schedule with the dose of $1.4 \mathrm{mg} /$ $\mathrm{m}^{2}$ in this study.

\section{Assessments}

The primary endpoint of this study was clinical benefit rate (CBR, defined as the proportion of patients who achieved complete response, partial response, or stable disease for $\geq 6$ months) based on RECIST v. 1.1. Secondary endpoints included time to treatment failure (TTF), OS, and safety. TTF was defined as the time from initiation of eribulin to treatment discontinuation for any reason, including disease progression, treatment toxicity, patient preference, or death caused by treatment. OS was calculated as the time from the initiation of eribulin until death from any cause. For safety, adverse events were recorded and graded according to the National Cancer Institute Common Terminology Criteria for Adverse Events (version 4.0, Japanese version) [18], and were coded according to Medical Dictionary for Regulatory Activities, Japanese version [19]. In addition, ad hoc analyses were performed: factors influencing the schedule modification of eribulin to bi-weekly administration were evaluated, and subgroup analyses were performed in elderly (aged $\geq 65$ years) and younger (aged $<65$ years) patients.

The study protocol was approved by local institutional review boards and ethics committees. This study was conducted in accordance with the Japanese Guidelines for Clinical Research of the Ministry of Health, Labour and Welfare and the Declaration of Helsinki, as well as other applicable regulatory requirements. All participants provided written informed consent prior to the study entry. The present study has been registered with the University Hospital Medical Information Network (UMIN) Center (ID: UMIN 000008491) [20].

\section{Statistical methods}

The sample size was calculated to evaluate the null hypothesis that the true CBR was $15 \%$ and the alternative hypothesis that the CBR was $\geq 30 \%$, with a type I error level of 0.1 and type II error level of 0.20 . The minimum target sample size was set at $N=70$ and we aimed to recruit 80 patients with the consideration that $\sim 10 \%$ of the recruited patients would not be evaluable. In the phase II clinical trial of eribulin in Japan, patients had a CBR of $27.5 \%$ with a median of 3 prior chemotherapy sessions. In addition, $33.3 \%$ of the patients could not be administered eribulin on day 8 of cycle 1 because of myelosuppression. Based on these findings, we assumed that $50 \%$ of the patients would not be eligible for eribulin on day 8 of cycle 1 in clinical practice.

The data cut-off date was February 25, 2016. The efficacy data were assessed in the eligible population who received at least one dose of the study drug and had evaluable efficacy data (full analysis set; FAS). The CBR and two-sided $95 \%$ CIs were calculated based on binominal distribution. TTF and OS (estimated median with $95 \% \mathrm{CI}$ ) were calculated using Kaplan-Meier methods. The safety data were assessed in the eligible population who received at least one dose of the study drug (safety population), which were presented by descriptive analyses. SAS software (version 9.4) (SAS Institute Inc., Cary, NC, USA) was used for all analyses.

\section{Results}

We conducted a phase II, prospective, non-randomized, open-label, multicenter study at 16 sites in Japan. A total of 88 patients were recruited in the study between July 2012 and April 2014. All 88 patients received at least one dose of eribulin. A total of 82 patients were eligible for efficacy analyses (FAS); 40 patients continued the standard eribulin therapy (standard group) and the remaining 42 patients required schedule modification (bi-weekly group) (Fig. 1).

The baseline characteristics of patients are summarized in Table 1. Although patients in bi-weekly group were intolerant to standard regimens, the characteristics and medical history were similar between the two groups.

Eribulin was administered for a median of 73.0 days (range 14-365) in the overall population, 77.0 days (range 35-322) in the standard group, and 87.0 days (range 28-365) in the bi-weekly group. Mean relative dose intensity was 
$76.9 \%$ in the overall population, $90.9 \%$ in the standard group, and $62.7 \%$ in the bi-weekly group when the dose intensity of $0.93 \mathrm{mg} / \mathrm{m}^{2} /$ week was considered as $100 \%$.

\section{Efficacy outcomes}

The ORR and CBR were 19.3\% (95\% CI 11.7-29.1) and $26.1 \%$ (95\% CI 17.3-36.6) in the overall population, $20.0 \%$ (95\% CI 9.1-35.6) and 25.0\% (95\% CI 12.7-41.2) in the standard group, and $21.4 \%$ (95\% CI 10.3-36.8) and $31.0 \%$ (95\% CI 17.6-47.1) in the bi-weekly group, respectively (Table 2).

The median TTF and OS were 77 days (95\% CI 70-95) and 427 days (95\% CI 300-701) in the overall population, 75 days (95\% CI 69-119) and 412 days (95\% CI 275-713) in the standard group, and 81.5 days (95\% CI 73-107) and 523 days (95\% CI 350-828) in the bi-weekly group (Table 2 ).

\section{Safety outcomes}

In the overall population, the most common adverse events with eribulin were leukopenia (83.0\%), neutropenia (77.3\%), anemia (58.0\%), fatigue (56.8\%), and alopecia (56.8\%) (Table 3). The most common grade 3 and 4 AEs were neutropenia (59.1\%) and leukopenia (45.5\%). In the bi-weekly group, the incidence rates of leukopenia and neutropenia of any grade were 95.2 and $92.9 \%$, respectively, whereas in the standard group, the corresponding incidence rates were 72.5 and $62.5 \%$, respectively. Febrile neutropenia was observed in one $(2.5 \%)$ patient in the standard group and five (11.9\%) patients in the bi-weekly group (Table 3 ).

Among patients in the bi-weekly group, grade 3/4 neutropenia was the major adverse event leading to schedule modification and reported in 34 patients $(81.0 \%)$ (Table 4$)$. Grade 3/4 leukopenia was reported in 22 patients (52.4\%). After transition to the bi-weekly schedule, grade 3/4 neutropenia reduced to $61.9 \%$ and grade $3 / 4$ leukopenia to $26.2 \%$. The frequencies of grade $3 / 4$ neutropenia and leukopenia during treatment cycles are shown in Fig. 2. By the modification of dosing schedule, the frequencies of these toxicities were dramatically decreased and the low incidences were maintained thereafter. Only $2(4.8 \%)$ patients experienced febrile neutropenia after schedule modification. The adverse events leading to discontinuation of eribulin were neutropenia (6 patients; $14.3 \%$ ) and peripheral sensory neuropathy (1 patient; $2.4 \%$ ) in the bi-weekly group (data not shown). No patients discontinued the study due to adverse event(s) in the standard group.

\section{Ad hoc analyses}

In the ad hoc analyses, we determined the association of dosing schedule modification with the baseline characteristics
Fig. 1 Patient disposition. FAS full analysis set

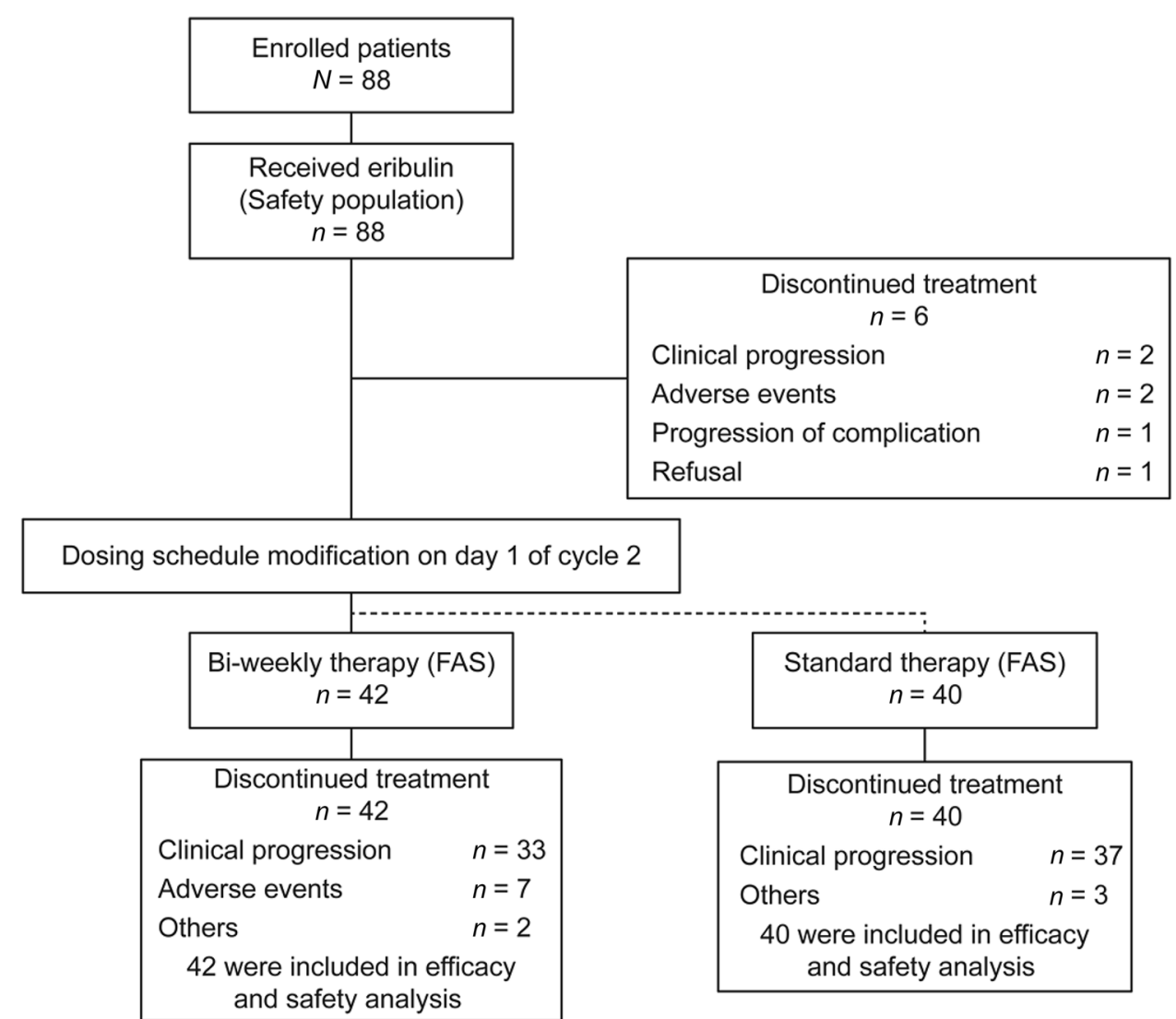


Table 1 Baseline characteristics of patients

\begin{tabular}{|c|c|c|c|}
\hline & $\begin{array}{l}\text { Overall } \\
N=88\end{array}$ & $\begin{array}{l}\text { Standard } \\
N=40\end{array}$ & $\begin{array}{l}\text { Bi-weekly } \\
N=42\end{array}$ \\
\hline $\begin{array}{l}\text { Median age, (range) } \\
\text { years }\end{array}$ & $59.5(37-80)$ & $61.0(37-80)$ & $60.0(40-77)$ \\
\hline \multicolumn{4}{|c|}{ ECOG performance status, $n(\%)$} \\
\hline 0 & $59(67.0)$ & $26(65.0)$ & $30(71.4)$ \\
\hline 1 & $28(31.8)$ & $14(35.0)$ & $12(28.6)$ \\
\hline 2 & $1(1.1)$ & 0 & 0 \\
\hline ER/PgR positive, $n(\%)$ & $54(61.4)$ & $23(57.5)$ & $27(64.3)$ \\
\hline Triple-negative, $n(\%)$ & $34(38.6)$ & $17(42.5)$ & $15(35.7)$ \\
\hline \multicolumn{4}{|c|}{ No. of chemotherapies for MBC, $n(\%)$} \\
\hline 0 & $15(17.0)$ & $5(12.5)$ & $9(21.4)$ \\
\hline 1 & $23(26.1)$ & $14(35.0)$ & $8(19.0)$ \\
\hline 2 & $27(30.7)$ & $10(25.0)$ & $14(33.3)$ \\
\hline 3 & $23(26.1)$ & $11(27.5)$ & $11(26.2)$ \\
\hline $\begin{array}{l}\text { No. of prior chemother- } \\
\text { apy regimens (median) }\end{array}$ & 2 & 2 & 2 \\
\hline \multicolumn{4}{|c|}{ Prior chemotherapy for $\mathrm{MBC}^{\mathrm{a}}, n(\%)$} \\
\hline FEC & $26(29.5)$ & $10(25.0)$ & $13(31.0)$ \\
\hline $\begin{array}{l}\text { Paclitaxel + bevaci- } \\
\text { zumab }\end{array}$ & $26(29.5)$ & $13(32.5)$ & $10(23.8)$ \\
\hline nab-Paclitaxel & $12(13.6)$ & $3(7.5)$ & $8(19.0)$ \\
\hline Paclitaxel & $13(14.8)$ & $7(17.5)$ & $6(14.3)$ \\
\hline S-1 & $12(13.6)$ & $4(10.0)$ & $6(14.3)$ \\
\hline $\mathrm{XC}$ & $11(12.5)$ & $5(12.5)$ & $6(14.3)$ \\
\hline Capecitabine & $15(17.0)$ & $9(22.5)$ & $5(11.9)$ \\
\hline Docetaxel & $6(6.8)$ & $4(10.0)$ & $2(4.8)$ \\
\hline Prior surgery, $n(\%)$ & $79(89.8)$ & $36(90.0)$ & $37(88.1)$ \\
\hline $\begin{array}{l}\text { Prior radiation therapy, } \\
n(\%)\end{array}$ & $29(33.0)$ & $10(25.0)$ & $15(35.7)$ \\
\hline \multicolumn{4}{|c|}{ Most common metastatic sites ${ }^{\mathrm{a}}, n(\%)$} \\
\hline Liver & $47(53.4)$ & $21(52.5)$ & $24(57.1)$ \\
\hline Bone & $41(46.6)$ & $22(55.0)$ & $18(42.9)$ \\
\hline Lymph nodes & $24(27.3)$ & $10(25.0)$ & $12(28.6)$ \\
\hline Lung & 19 (21.6) & $9(22.5)$ & $7(16.7)$ \\
\hline Axilla & $14(15.9)$ & $6(15.0)$ & $7(16.7)$ \\
\hline $\begin{array}{l}\text { No. of metastatic sites, } \\
\text { median }\end{array}$ & 1 & 1 & 2 \\
\hline
\end{tabular}

Overall population $=$ safety population $=88$ patients

6 patients were excluded ( 2 due to progressive disease, 2 due to adverse events, 1 due to complication, 1 due to refusal)

Standard therapy group $=$ efficacy analysis population $=40$ patients Biweekly therapy group $=$ efficacy analysis population $=42$ patients ECOG Eastern Cooperative Oncology Group, ER estrogen receptor, $P g R$ progesterone receptor, $F E C$ 5-FU + epirubicin + cyclophosphamide, $M B C$ metastatic breast cancer, $S 1$ tegafur/gimeracil/oteracil potassium, $X C$ capecitabine + cyclophosphamide

${ }^{a}$ Data shown are for $>10 \%$ of patients

including age, neutrophil count (Table 5), presence of liver metastases, aspartate aminotransferase level, alanine aminotransferase level, and blood albumin level (data not
Table 2 Response rate

\begin{tabular}{llll}
\hline & $\begin{array}{l}\text { Overall } \\
N=88\end{array}$ & $\begin{array}{l}\text { Standard } \\
N=40\end{array}$ & $\begin{array}{l}\text { Bi-weekly } \\
N=42\end{array}$ \\
\hline Best overall response, $n(\%)$ & & & \\
Complete response & $2(2.3)$ & $1(2.5)$ & $1(2.4)$ \\
Partial response & $15(17.0)$ & $7(17.5)$ & $8(19.0)$ \\
Stable disease & $23(26.1)$ & $9(22.5)$ & $13(31.0)$ \\
Stable disease $\geq 6$ months & $6(6.8)$ & $2(5.0)$ & $4(9.5)$ \\
Progressive disease & $37(42.0)$ & $21(52.5)$ & $15(35.7)$ \\
Not evaluable & $5(5.7)$ & 0 & $1(2.4)$ \\
Objective response rate, $n(\%)$ & $17(19.3)$ & $8(20.0)$ & $9(21.4)$ \\
95\% CI & $11.7-29.1$ & $9.1-35.6$ & $10.3-36.8$ \\
Clinical benefit rate, $n(\%)$ & $23(26.1)$ & $10(25.0)$ & $13(31.0)$ \\
95\% CI & $17.3-36.6$ & $12.7-41.2$ & $17.6-47.1$ \\
Time to treatment failure, days & 77 & 75 & 81.5 \\
95\% CI & $70-95$ & $69-119$ & $73-107$ \\
Overall survival, days & 427 & 412 & 523 \\
95\% CI & $300-701$ & $275-713$ & $350-828$ \\
\hline
\end{tabular}

CI confidence interval

shown). However, these were not significantly associated with the schedule modification of eribulin (Table 5).

In elderly patients (aged $\geq 65$ years), the CBR (Table 5) and ORR (data not shown) were 35.7 and $21.4 \%$ in the standard group, and 33.3 and $25.0 \%$ in the bi-weekly group, respectively. On the other hand, in younger patients (aged $<65$ years), the CBR and ORR were 19.2 and 19.2\% in the standard group, and 30.0 and $20.0 \%$ in the bi-weekly group, respectively. Most likely, elderly patients may be susceptible to myelosuppression and be unable to continue on the standard schedule of eribulin. Bi-weekly eribulin therapy is an effective and safe option for elderly patients.

\section{Discussion}

Management of eribulin dose and schedule is a key issue to decrease toxicity and increase efficacy. Among patients who required schedule modification, the majority of patients (76.2\%) needed it due to neutropenia. Although leukopenia and neutropenia were more frequently observed in patients who required schedule modification compared with those who continued the standard regimen of eribulin, before and after switching to bi-weekly administration, incidence of grade $3 / 4$ leukopenia decreased from 52.4 to $26.2 \%$ and grade $3 / 4$ neutropenia decreased from 81.0 to $61.9 \%$. By modification of dosing schedule, the frequencies of these toxicities were dramatically decreased in the second cycle and thereafter. There was no incidence of grade 4 of leukopenia and neutropenia after 4 cycles in bi-weekly therapy, suggesting that the majority of patients transferred to the 
Table 3 Adverse events

\begin{tabular}{|c|c|c|c|c|c|c|c|c|c|}
\hline & \multicolumn{3}{|c|}{ Overall $(N=88)$} & \multicolumn{3}{|c|}{ Standard $(N=40)$} & \multicolumn{3}{|c|}{ Bi-weekly $(N=42)$} \\
\hline & Any grade & Grade 3 & Grade 4 & Any grade & Grade 3 & Grade 4 & Any grade & Grade 3 & Grade 4 \\
\hline \multicolumn{10}{|l|}{ Hematologic events, $n(\%)$} \\
\hline Leukopenia & $73(83.0)$ & $32(36.4)$ & $8(9.1)$ & $29(72.5)$ & $9(22.5)$ & $2(5.0)$ & $40(95.2)$ & $22(52.4)$ & $3(7.1)$ \\
\hline Neutropenia & $68(77.3)$ & $28(31.8)$ & $24(27.3)$ & $25(62.5)$ & $9(22.5)$ & $3(7.5)$ & $39(92.9)$ & $18(42.9)$ & $19(45.2)$ \\
\hline Anemia & $51(58.0)$ & $3(3.4)$ & 0 & $24(60.0)$ & $3(7.5)$ & 0 & $23(54.8)$ & 0 & 0 \\
\hline Thrombocytopenia & $16(18.2)$ & $3(3.4)$ & 0 & $9(22.5)$ & $2(5.0)$ & 0 & $5(11.9)$ & 0 & 0 \\
\hline Febrile neutropenia & $7(8.0)$ & $7(7.0)$ & 0 & $1(2.5)$ & $1(2.5)$ & 0 & $5(11.9)$ & $5(11.9)$ & 0 \\
\hline \multicolumn{10}{|l|}{ Non-hematologic events, $n(\%)$} \\
\hline AST increased & $45(51.1)$ & $3(3.4)$ & 0 & $18(45.0)$ & 0 & 0 & $24(57.1)$ & $2(4.8)$ & 0 \\
\hline Alopecia & $50(56.8)$ & 0 & 0 & $26(65.0)$ & 0 & 0 & $23(54.8)$ & 0 & 0 \\
\hline Peripheral sensory neuropathy & $42(47.7)$ & $1(1.1)$ & 0 & $18(45.0)$ & 0 & 0 & $22(52.4)$ & $1(2.4)$ & 0 \\
\hline Fatigue & $50(56.8)$ & $5(5.7)$ & 0 & $24(60.0)$ & $4(10.0)$ & 0 & $22(52.4)$ & $1(2.4)$ & 0 \\
\hline Malaise & $46(52.3)$ & 0 & 0 & $22(55.0)$ & 0 & 0 & $19(45.2)$ & 0 & 0 \\
\hline ALT increased & $37(42.0)$ & $2(2.3)$ & 0 & $17(42.5)$ & 0 & 0 & $18(42.9)$ & $2(4.8)$ & 0 \\
\hline Nausea & $32(36.4)$ & $1(1.1)$ & 0 & $15(37.5)$ & 0 & 0 & $17(40.5)$ & $1(2.4)$ & 0 \\
\hline Dysgeusia & $32(36.4)$ & 0 & 0 & $18(45.0)$ & 0 & 0 & $12(28.6)$ & 0 & 0 \\
\hline Mucositis oral & $21(23.9)$ & $1(1.1)$ & 0 & $9(22.5)$ & 0 & 0 & $11(26.2)$ & $1(2.4)$ & 0 \\
\hline Pain & $24(27.3)$ & $3(3.4)$ & 0 & $12(30.0)$ & 0 & 0 & $9(21.4)$ & $2(4.8)$ & 0 \\
\hline Constipation & $18(20.5)$ & 0 & 0 & $8(20.0)$ & 0 & 0 & $8(19.0)$ & 0 & 0 \\
\hline Peripheral motor neuropathy & $14(15.9)$ & $2(2.3)$ & 0 & $4(10.0)$ & 0 & 0 & $8(19.0)$ & $1(2.4)$ & 0 \\
\hline Nail discoloration & $11(12.5)$ & 0 & 0 & $5(12.5)$ & 0 & 0 & $6(14.3)$ & 0 & 0 \\
\hline Edema limbs & $9(10.2)$ & 0 & 0 & $4(10.0)$ & 0 & 0 & $5(11.9)$ & 0 & 0 \\
\hline Rash maculo-papular & $4(4.5)$ & $1(1.1)$ & 0 & 0 & 0 & 0 & $4(9.5)$ & $1(2.4)$ & 0 \\
\hline Vomiting & $5(5.7)$ & 0 & 0 & $2(5.0)$ & 0 & 0 & $3(7.1)$ & 0 & 0 \\
\hline Pharyngitis & $4(4.5)$ & 0 & 0 & $1(2.5)$ & 0 & 0 & $3(7.1)$ & 0 & 0 \\
\hline Diarrhea & $5(5.7)$ & 0 & 0 & $3(7.5)$ & 0 & 0 & $2(4.8)$ & 0 & 0 \\
\hline Blood bilirubin increased & $7(8.0)$ & 0 & 0 & $3(7.5)$ & 0 & 0 & $2(4.8)$ & 0 & 0 \\
\hline Nail loss & $1(1.1)$ & 0 & 0 & 0 & 0 & 0 & $1(2.4)$ & 0 & 0 \\
\hline Skin hyperpigmentation & $3(3.4)$ & 0 & 0 & $2(5.0)$ & 0 & 0 & $1(2.4)$ & 0 & 0 \\
\hline Creatinine increased & $9(10.2)$ & $2(2.3)$ & 0 & $7(17.5)$ & $2(5.0)$ & 0 & $1(2.4)$ & 0 & 0 \\
\hline
\end{tabular}

$A L T$ alanine aminotransferase, $A S T$ aspartate aminotransferase

bi-weekly regimen could continue eribulin therapy safely for 8 courses. Furthermore, the frequency of hospital visits was $20 \%$ lower with the bi-weekly schedule as compared with the standard schedule.

The efficacy results of patients who required schedule modification were comparable to those of patients who continued the standard eribulin regimen. In addition, our ad hoc analyses demonstrated that the tolerability of eribulin was comparable between patients aged $<65$ years and those aged $\geq 65$ years. Thus, eribulin may also be beneficial for elderly patients.

The efficacy results with the modified bi-weekly schedule in the present study were comparable to those of a previous phase II study conducted in Japan with the standard regimen [13]. CBR and ORR were 31.0 and $21.4 \%$ in patients who received bi-weekly therapy of eribulin, compared to 27.5 and $21.3 \%$, in patients on the standard regimen in the previous phase II study [13]. The median OS was also similar and was 523 days (17.2 months) in the present study and 11.1 months in the previous phase II study [13]. In addition, the adverse events observed in the present study were consistent with those in previous phase II/III studies conducted globally or in Japan [10-13, 21-25], and no new safety signals were detected. Hematological toxicities are common with eribulin. In the present study, the majority of patients experienced hematologic adverse events, including neutropenia and leucopenia. In the overall population, grade $3 / 4$ neutropenia was observed in $59.1 \%$, and grade $3 / 4$ leukopenia was observed in $45.5 \%$ of patients, figures which are relatively low compared to the previous phase II study conducted in Japan (95.1 and $74.1 \%$, respectively) [13]. Moreover, the incidence of grade 3/4 febrile neutropenia in the present study was $8.0 \%$, which was lower than the $13.6 \%$ 
Table 4 Adverse events: before/ after schedule modification (bi-weekly group; $N=42$ )

\begin{tabular}{|c|c|c|c|c|c|c|}
\hline \multirow[t]{2}{*}{ Adverse events, $n(\%)$} & \multicolumn{3}{|c|}{ Before schedule modification } & \multicolumn{3}{|c|}{ After schedule modification } \\
\hline & Any grade & Grade 3 & Grade 4 & Any grade & Grade 3 & Grade 4 \\
\hline Leukopenia & $39(92.9)$ & $19(45.2)$ & $3(7.1)$ & $34(81.0)$ & $11(26.2)$ & 0 \\
\hline Neutropenia & $39(92.9)$ & $19(45.2)$ & $15(35.7)$ & $35(83.3)$ & $18(42.9)$ & $8(19.0)$ \\
\hline Anemia & $21(50.0)$ & 0 & 0 & $22(52.4)$ & 0 & 0 \\
\hline AST increased & $21(50.0)$ & $1(2.4)$ & 0 & $22(52.4)$ & $2(4.8)$ & 0 \\
\hline Hypoalbuminemia & $21(50.0)$ & 0 & 0 & $21(50.0)$ & 0 & 0 \\
\hline Fatigue & $20(47.6)$ & 0 & 0 & $20(47.6)$ & $1(2.4)$ & 0 \\
\hline Peripheral sensory neuropathy & $19(45.2)$ & 0 & 0 & $22(52.4)$ & $1(2.4)$ & 0 \\
\hline Alopecia & $18(42.9)$ & 0 & 0 & $23(54.8)$ & 0 & 0 \\
\hline Malaise & $17(40.5)$ & 0 & 0 & $19(45.2)$ & 0 & 0 \\
\hline ALT increased & $15(35.7)$ & $1(2.4)$ & 0 & $12(28.6)$ & $2(4.8)$ & 0 \\
\hline Nausea & $12(28.6)$ & 0 & 0 & $13(31.0)$ & $1(2.4)$ & 0 \\
\hline Dysgeusia & $10(23.8)$ & 0 & 0 & $9(21.4)$ & 0 & 0 \\
\hline Peripheral motor neuropathy & $8(19.0)$ & 0 & 0 & $8(19.0)$ & $1(2.4)$ & 0 \\
\hline Pain & $7(16.7)$ & $1(2.4)$ & 0 & $9(21.4)$ & $2(4.8)$ & 0 \\
\hline Mucositis oral & $6(14.3)$ & $1(2.4)$ & 0 & $11(26.2)$ & 0 & 0 \\
\hline Constipation & $4(9.5)$ & 0 & 0 & $7(16.7)$ & 0 & 0 \\
\hline Thrombocytopenia & $4(9.5)$ & 0 & 0 & $2(4.8)$ & 0 & 0 \\
\hline Febrile neutropenia & $3(7.1)$ & $3(7.1)$ & 0 & $2(4.8)$ & $2(4.8)$ & 0 \\
\hline Blood bilirubin increased & 0 & 0 & 0 & $2(4.8)$ & 0 & 0 \\
\hline Creatinine increased & 0 & 0 & 0 & $1(2.4)$ & 0 & 0 \\
\hline
\end{tabular}

$A L T$ alanine aminotransferase, $A S T$ aspartate aminotransferase

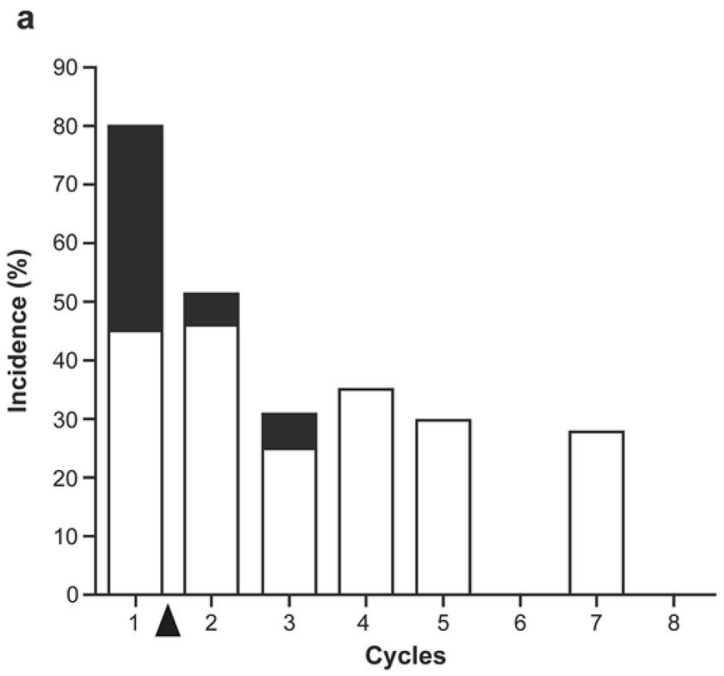

Fig. 2 Frequency of grade 3/4 neutropenia and leukopenia during treatment cycles in bi-weekly group. a Neutropenia; b leukopenia. White and black columns represent grades 3 and 4, respectively.

in the previous phase II study [13]. Only 2 (4.8\%) patients experienced febrile neutropenia after schedule modification.

To our knowledge, this is the first study investigating safety and effectiveness of bi-weekly administration of eribulin in patients with $\mathrm{MBC}$; however, this study has some limitations. This was a non-randomized study: the patients

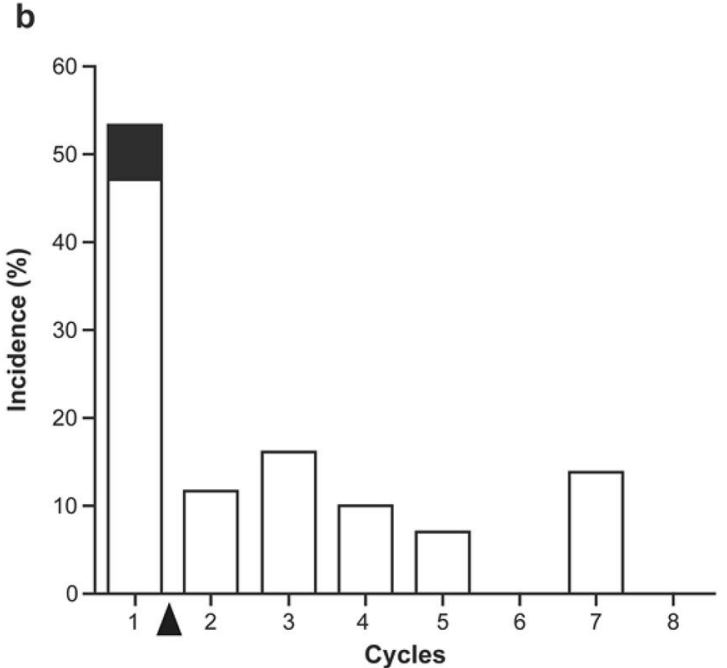

Black arrow indicates the timing of the schedule modification based on the adverse events in the first cycle

were not randomly assigned to the standard group and biweekly group and the patients in the bi-weekly group were those who were unable to continue the standard regimen of eribulin. Moreover, sample sizes of both groups (standard schedule and bi-weekly schedule) as well as subgroups 
Table 5 Subgroup analyses (CBR)

\begin{tabular}{|c|c|c|c|c|c|c|}
\hline & $N$ & Overall $(N=88)$ & $N$ & Standard $(N=40)$ & $N$ & Bi-weekly $(N=42)$ \\
\hline All & 88 & $23(26.1)$ & 40 & $10(25.0)$ & 42 & $13(31.0)$ \\
\hline \multicolumn{7}{|c|}{ Hormone receptor status (ER and/or PgR) } \\
\hline Positive & 54 & 16 (29.6) & 23 & $7(30.4)$ & 27 & $9(33.3)$ \\
\hline Negative & 34 & $7(20.6)$ & 17 & $3(17.6)$ & 15 & $4(26.7)$ \\
\hline \multicolumn{7}{|c|}{ No. of chemotherapy regimens for advanced or MBC } \\
\hline 0 & 15 & $7(46.7)$ & 5 & $2(40.0)$ & 9 & $5(55.6)$ \\
\hline 1 & 23 & $4(17.4)$ & 14 & $2(14.3)$ & 8 & $2(25.0)$ \\
\hline 2 & 27 & $7(25.9)$ & 10 & $4(40.0)$ & 14 & $3(21.4)$ \\
\hline 3 & 23 & $5(21.7)$ & 11 & $2(18.2)$ & 11 & $3(27.3)$ \\
\hline \multicolumn{7}{|c|}{ Relative dose intensity } \\
\hline$>75 \%$ & 50 & $10(20.0)$ & 36 & $9(25.0)$ & 8 & $1(12.5)$ \\
\hline$<75 \%$ & 38 & $13(34.2)$ & 4 & $1(25.0)$ & 34 & $12(35.3)$ \\
\hline \multicolumn{7}{|c|}{ Neutropenia (cycle 1, day 8) } \\
\hline$>1000$ & 57 & $15(26.3)$ & 39 & $10(25.6)$ & 14 & $5(35.7)$ \\
\hline$<1000$ & 30 & $8(26.7)$ & 0 & $0(0.0)$ & 28 & $8(28.6)$ \\
\hline \multicolumn{7}{|l|}{ Age } \\
\hline$\geq 65$ & 26 & $9(34.6)$ & 14 & $5(35.7)$ & 12 & $4(33.3)$ \\
\hline$<65$ & 56 & $15(26.8)$ & 26 & $5(19.2)$ & 30 & $9(30.0)$ \\
\hline
\end{tabular}

$C B R$ clinical benefit rate, $E R$ estrogen receptor, $P g R$ progesterone receptor, $M B C$ metastatic breast cancer were small. Therefore, the findings should be interpreted with caution.

In conclusion, this phase II study involving patients with MBC demonstrated that bi-weekly administration of eribulin in patients who were intolerant to the standard regimen of eribulin had antitumor activity comparable to the standard therapy. Since the risk of adverse events was reduced after switching to bi-weekly regimen in the patients who were not tolerant to the standard regimen of eribulin, bi-weekly administration of eribulin might be an alternative option for such patients. In addition, the bi-weekly schedule may contribute to the future exploration of combination therapy of eribulin with other cancer agents.

Acknowledgements The authors would like to express their deepest gratitude to the patients who participated in this trial and their family members, in addition to the investigators and all staff members at the study sites for their contribution to the study. The authors acknowledge Miss Yu Fujishiro for data collection and management. Medical writing and editorial assistance for the preparation of this manuscript was provided by Dr. Koki Yamashita on behalf of CACTUS Communications and was funded by Eisai Co., Ltd. (Tokyo, Japan).

The results of the present study have been reported previously at the 2015 Annual Meeting of the American Society for Clinical Oncology.

\section{Compliance with ethical standards}

Conflict of interest This is an investigator-initiated clinical study; this study was not supported by any industry funding, nor requested by any organization. HK received honoraria from Chugai, AstraZeneca, Eisai, and Kyowa Kirin. SO, TN, TY, KW, FH, YS, KH, N Matsunami, YH, MT, MM, TM, MS, MI, SM, and N Masuda have nothing to disclose.
Open Access This article is distributed under the terms of the Creative Commons Attribution 4.0 International License (http://creativeco mmons.org/licenses/by/4.0/), which permits unrestricted use, distribution, and reproduction in any medium, provided you give appropriate credit to the original author(s) and the source, provide a link to the Creative Commons license, and indicate if changes were made.

\section{References}

1. Cortes J, Vidal M. Beyond taxanes: the next generation of microtubule-targeting agents. Breast Cancer Res Treat. 2012;133:821-30.

2. Jain S, Cigler T. Eribulin mesylate in the treatment of metastatic breast cancer. Biologics. 2012;6:21-9.

3. Swami U, Chaudhary I, Ghalib MH, Goel S. Eribulin-a review of preclinical and clinical studies. Crit Rev Oncol Hematol. 2012;81:163-84.

4. Inoue K, Saito T, Okubo K, Kimizuka K, Yamada H, Sakurai T, et al. Phase II clinical study of eribulin monotherapy in Japanese patients with metastatic breast cancer who had well-defined taxane resistance. Breast Cancer Res Treat. 2016;157:295-305.

5. US Food and Drug Administration. Highlights of prescribing information: Halaven. 2010. http://www.accessdata.fda.gov/drugs atfda_docs/label/2010/201532lbl.pdf. Accessed 01 Mar 2017.

6. Kok VC. Eribulin in the management of advanced breast cancer: implications of current research findings. Breast Cancer (Auckl). 2015;9:109-15.

7. Ro J, Cheng FT, Sriuranpong V, Villalon A, Smruti BK, Tsang $\mathrm{J}$, et al. Patient management with eribulin in metastatic breast cancer: a clinical practice guide. J Breast Cancer. 2016;19:8-17.

8. Eisai: Halaven ${ }^{\circledR}$ receives approval in Japan for the treatment of inoperable and recurrent breast cancer. 2011. http://www.eisai .com/news/enews201133pdf.pdf. Accessed 7 June 2016.

9. Watanabe J. Eribulin monotherapy improved survivals in patients with ER-positive HER2-negative metastatic breast cancer in the real world: a single institutional review. Springerplus. 2015;4:625. 
10. Cortes J, O’Shaughnessy J, Loesch D, Blum JL, Vahdat LT, Petrakova K, et al. Eribulin monotherapy versus treatment of physician's choice in patients with metastatic breast cancer (EMBRACE): a phase 3 open-label randomised study. Lancet. 2011;377:914-23.

11. Kaufman PA, Awada A, Twelves C, Yelle L, Perez EA, Velikova G, et al. Phase III open-label randomized study of eribulin mesylate versus capecitabine in patients with locally advanced or metastatic breast cancer previously treated with an anthracycline and a taxane. J Clin Oncol. 2015;33:594-601.

12. Twelves C, Cortes J, Vahdat L, Olivo M, He Y, Kaufman PA, et al. Efficacy of eribulin in women with metastatic breast cancer: a pooled analysis of two phase 3 studies. Breast Cancer Res Treat. 2014;148:553-61.

13. Aogi K, Iwata H, Masuda N, Mukai H, Yoshida M, Rai Y, et al. A phase II study of eribulin in Japanese patients with heavily pretreated metastatic breast cancer. Ann Oncol. 2012;23:1441-8.

14. Reed E, Simmonds P, Haviland J, Corner J. Quality of life and experience of care in women with metastatic breast cancer: a cross-sectional survey. J Pain Symptom Manage. 2012;43:747-58.

15. Cortes J, Hudgens S, Twelves C, Perez EA, Awada A, Yelle $\mathrm{L}$, et al. Health-related quality of life in patients with locally advanced or metastatic breast cancer treated with eribulin mesylate or capecitabine in an open-label randomized phase 3 trial. Breast Cancer Res Treat. 2015;154:509-20.

16. Oken MM, Creech RH, Tormey DC, Horton J, Davis TE, McFadden ET, et al. Toxicity and response criteria of the Eastern Cooperative Oncology Group. Am J Clin Oncol. 1982;5:649-55.

17. Eisenhauer EA, Therasse P, Bogaerts J, Schwartz LH, Sargent D, Ford R, et al. New response evaluation criteria in solid tumours: revised RECIST guideline (version 1.1). Eur J Cancer. 2009;45:228-47.

18. Japan Clinical Oncology Group. Common Terminology Criteria for Adverse Events (CTCAE) Version 4.0. 2009. http://www.jcog. jp/doctor/tool/CTCAEv4J_20130409.pdf. Accessed 28 June 2016.
19. MedDRA Japanese Maintenance Organization. Medical dictionary for regulatory activities, Japanese version. 2010. https://www. pmrj.jp/jmo/php/indexj.php. Accessed 9 June 2016.

20. University Hospital Medical Information Network (UMIN) Center ID: UMIN000008491. Japanese study to evaluate the utility of eribulin dose and schedule in treatment for advanced breast cancer patients (JUST-STUDY). https://upload.umin.ac.jp/cgi-open-bin/ ctr/ctr.cgi?function $=$ brows \&action $=$ brows \& type $\% 20=$ summa ry\&recptno $=$ R000009978\&language $=\mathrm{J}$. Accessed 28 June 2016.

21. Cortes J, Vahdat L, Blum JL, Twelves C, Campone M, Roché H, et al. Phase II study of the halichondrin B analog eribulin mesylate in patients with locally advanced or metastatic breast cancer previously treated with an anthracycline, a taxane, and capecitabine. J Clin Oncol. 2010;28:3922-8.

22. McIntyre K, O'Shaughnessy J, Schwartzberg L, Glück S, Berrak E, Song JX, et al. Phase 2 study of eribulin mesylate as firstline therapy for locally recurrent or metastatic human epidermal growth factor receptor 2-negative breast cancer. Breast Cancer Res Treat. 2014; 146:321-8.

23. O'Shaughnessy J, McIntyre K, Schwartzberg L, Wilks S, Puhalla $\mathrm{S}$, Berrak E, et al. Impact of prior anthracycline or taxane use on eribulin effectiveness as first-line treatment for metastatic breast cancer: results from two phase 2 , multicenter, single-arm studies. Springerplus. 2015;4:532.

24. Tan AR, Rubin EH, Walton DC, Shuster DE, Wong YN, Fang F, et al. Phase I study of eribulin mesylate administered once every 21 days in patients with advanced solid tumors. Clin Cancer Res. 2009:15:4213-9.

25. Vahdat LT, Pruitt B, Fabian CJ, Rivera RR, Smith DA, TanChiu E, et al. Phase II study of eribulin mesylate, a halichondrin B analog, in patients with metastatic breast cancer previously treated with an anthracycline and a taxane. J Clin Oncol. 2009;27:2954-61.

\section{Affiliations}

\section{Shoichiro Ohtani ${ }^{1} \cdot$ Takahiro Nakayama $^{2} \cdot$ Tetsuhiro Yoshinami $^{2} \cdot$ Ken-ichi Watanabe $^{3} \cdot$ Fumikata Hara $^{4}$. Yasuaki Sagara ${ }^{5} \cdot$ Hidetoshi Kawaguchi $^{6} \cdot$ Kenji Higaki $^{7} \cdot$ Nobuki Matsunami $^{8} \cdot$ Yoshie Hasegawa $^{9}$.

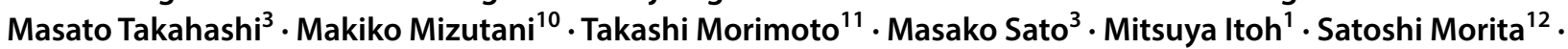 Norikazu Masuda ${ }^{10}$}

1 Division of Breast Surgery, Hiroshima City Hiroshima Citizens Hospital, 7-33 Motomachi, Naka-ku, Hiroshima-shi, Hiroshima 730-8518, Japan

2 Osaka Medical Center for Cancer and Cardiovascular Diseases, 1-3-3 Nakamichi, Higashinari-ku, Osaka-shi, Osaka, Japan

3 NHO Hokkaido Cancer Center, 3-54 4-jo 2-chome Kikusui Shiroishi-ku, Sapporo-shi, Hokkaido, Japan

4 Department of Breast Oncology, NHO Shikoku Cancer Center, 160 Kou, Minamiumemoto-machi, Matsuyama-shi, Ehime, Japan

5 Division of Breast Surgery, Hakuaikai Medical Corp. Sagara Hospital, 3-28 Matsubara-cho, Kagoshima-shi, Japan

6 Matsuyama Red Cross Hospital, 1 Bunkyo-cho, Matsuyama-shi, Ehime, Japan
7 Higaki Breast Clinic, 8-23 Hondori, Naka-ku, Hiroshima-shi, Hiroshima, Japan

8 Osaka Rosai Hospital, 1179-3 Nagasonecho, Kita-ku, Sakai-shi, Osaka, Japan

9 Department of Breast Surgery, Hirosaki Municipal Hospital, 3-8-1 Omachi, Hirosaki-shi, Aomori, Japan

10 Department of Surgery, Breast Oncology, NHO Osaka National Hospital, Chu-ouku Hoenzaka 2-1-14, Osaka-shi, Osaka, Japan

11 Yao Municipal Hospital, 1-3-1 Ryuge-cho, Yao-shi, Osaka, Japan

12 Kyoto University Graduate School of Medicine, 54 Kawahara-cho, Shogoin, Sakyo-ku, Kyoto, Japan 